\title{
MeerGAL: the MeerKAT Galactic Plane Survey
}

\author{
M.A. Thompson*1, S. Goedhart ${ }^{2}$ P. Benaglia ${ }^{3}$, H. Beuther ${ }^{4}$, R. Blomme ${ }^{5}$, \\ A.C. Chrysostomou ${ }^{6}$, J.S. Clark ${ }^{7}$, C. Dickinson ${ }^{8}$, S. Ellingsen ${ }^{9}$, D. Fenech ${ }^{10}$, \\ L. Hindson ${ }^{1}$, H.J. van Langevelde ${ }^{11}$, S.N. Longmore ${ }^{12}$, G. MacLeod ${ }^{13}$, S. Molinari ${ }^{14}$, \\ R. Prinja ${ }^{10}$, C.R. Purcell ${ }^{15}$, I. Stevens ${ }^{16}$, G. Umana ${ }^{17}$, J.S. Urquhart ${ }^{18}$, \\ W. Vlemmings ${ }^{19}$, A.J. Walsh ${ }^{20}$, J. van der Walt ${ }^{21}$, A.Y. Yang ${ }^{1,22}$ A. Zijlstra ${ }^{8}$ \\ ${ }^{1}$ University of Hertfordshire, UK, E-mail: m.a.thompson@herts . ac .uk, ${ }^{2} S K A$ Africa, \\ Cape Town, South Africa, ${ }^{3}$,Instituto Argentino de Radioastronomia, Argentina ${ }^{4}$, Max Planck \\ Institut für Astronomie, Heidelberg, Germany, ${ }^{5}$, Royal Observatory of Belgium, Belgium, ${ }^{6}$, SKA \\ Organisation, UK, ${ }^{7}$, Open University, UK, ${ }^{8}$ University of Manchester, UK, ${ }^{9}$ University of \\ Tasmania, Australia, ${ }^{10}$ University College London, UK, ${ }^{11}$ Joint Institute for VLBI in Europe, The \\ Netherlands, ${ }^{12}$ Liverpool John Moores University, UK, ${ }^{13}$ Hartebeesthoek Radio Observatory, \\ South Africa, ${ }^{14}$ Istituto Nazionale di Astrofisica, Rome, Italy, ${ }^{15}$ Macquarie University, Australia, \\ ${ }^{16}$ University of Birmingham, UK, ${ }^{17}$ INAF, Catania Astrophysical Observatory, Italy, ${ }^{18}$ University \\ of Kent, UK, ${ }^{19}$ Chalmers University of Technology, Sweden, ${ }^{20}$ Curtin University, Australia, \\ ${ }^{21}$ North-West University, South Africa, ${ }^{22}$ National Astronomical Observatories, Beijing, China.
}

Radio surveys of the Milky Way galaxy have transformed our understanding of star formation and stellar evolution. However, due to strong dependence of "survey cost" on frequency most large area surveys have so far been carried out at low frequencies ( $\sim$ a few GHz). These surveys select against dense plasma as the free-free turnover frequency scales directly with electron density which means that there are significant biases against the detection of the youngest and densest HII regions, Young Stellar Objects, jets, winds and Planetary Nebulae. Here we describe the MeerKAT Large Project MeerGAL, which aims to address this issue by making the first sensitive high frequency, high resolution multi-epoch survey of the Galactic Plane. Together with its Northern Hemisphere sister project KuGARS (the Ku-band Galactic Reconnaissance Survey), MeerGAL will revolutionise the study of massive star formation and stellar evolution, Galactic structure, and variability.

MeerKAT Science: On the Pathway to the SKA,

25-27 May, 2016,

Stellenbosch, South Africa

${ }^{*}$ Speaker. 


\section{MeerGAL: a unique window on the steep spectrum, optically thick Galaxy}

Large area unbiased surveys are one of the cornerstones of astronomy, with an impact often far beyond their original expectations and conceived wavelength ranges. This is particularly true in radio astronomy where pulsars, radio galaxies, quasars and gravitational lenses are all serendipitous discoveries of radio sky surveys (1). For Galactic studies, radio surveys are particularly powerful as the Galaxy is largely transparent at radio wavelengths and so we are able to study objects across the Galaxy even at low Galactic latitude. Most radio surveys have so far been carried out at low frequency ( $v \leq 5 \mathrm{GHz}$ ), e.g. NVSS (2), SUMSS (3), MAGPIS (4), CGPS (5), CORNISH (6) and more recently THOR (7) and VLASS (8). The reason behind this is that high frequency radio surveys are expensive. The time required for a radio survey is the product of the number of pointings $N$ and the time $t$ required for each pointing to reach the desired flux density level $(S)$ of the survey. For a fixed aperture $N$ scales as $v^{2}$ and $t$ scales as $S^{-2}$. So for objects with negative or slowly falling spectral indices such as non-thermal or optically thin thermal emission $\left(S \propto v^{-0.7}\right.$ to $\left.v^{-0.1}\right)$ the "cost" of a fixed-aperture constant sensitivity survey scales as $v^{2.2}$ to $v^{3.4}$. So $1.4-5 \mathrm{GHz}$ surveys are the most effective way to find objects that are either non-thermal or optically thin at these frequencies (e.g. supernova remnants, synchrotron-dominated radio stars and compact/ultracompact HII regions).

However, low frequency surveys have a strong selection bias against objects whose spectra rise with frequency. One particular Galactic example is that of the hypercompact (HC) HII region, an extremely compact dense HII region 10 times smaller and 100 times more dense than the ultracompact (UC) HII region, its more well known immediate descendant (9). Because of their strong rising spectra $\left(S \propto v^{2}\right)$ HC HII regions went completely undetected during the many successful 5 $\mathrm{GHz}$ surveys of the Galaxy and have so far only been serendipitously discovered near ultracompact HII regions observed at high frequencies. The turnover frequency between the optically thick and thin regimes for thermal bremsstrahlung is essentially a linear function of electron density (10) and so this means that low frequency $(v \leq 5 \mathrm{GHz})$ surveys preferentially select against dense plasma $\left(n_{\mathrm{e}} \gtrsim 10^{5} \mathrm{~cm}^{-3}\right)$. This not only includes objects such as HC HII regions, but massive stellar winds, ionised jets from YSOs (11) and young planetary nebulae (12) - a significant bias against the youngest and densest objects in each class.

The lack of high angular resolution surveys in this frequency regime means that our knowledge of the overall properties of these objects, and indeed the global distribution of steep spectrum emission in the Galaxy is extremely poor. There are three major high frequency surveys covering the Galactic Plane (notwithstanding all-sky surveys by WMAP and Planck), the single-dish Nobeyama $10 \mathrm{GHz}$ (13) and (14) Green Bank 8 \& $14 \mathrm{GHz}$ surveys and the interferometric AT20G survey (15). All of these surveys are too shallow ( $1 \sigma$ depths of $42 \mathrm{mJy}-0.8 \mathrm{Jy}$ ) and/or of too low resolution (arcmin in the case of Nobeyama \& Langston surveys) to identify HC HII regions. These surveys have however been successful in uncovering tantalising hints of the high frequency population and its variability. The Langston et al survey reveals hints of a variable and transient population (16) and indeed, later interferometric studies of UC \& HC HII regions have shown that their variability is a powerful probe of the physics involved in the transition phase between Massive Young Stellar Object and HII region $(17 ; 18 ; 19)$. Multi-year epochs of radio variability offer a powerful way of measuring distances to \& core masses of planetary nebulae (20). Unexplained low frequency 
transients are beginning to be discovered (21), are there analogous high frequency examples? In addition, the high frequency bands are beginning to offer insight into new phases of the ISM, for example the population of small spinning grains revealed by their anomalous microwave emission $(22 ; 23 ; 24)$.

What is needed to investigate the Galactic high frequency population is a sensitive and uniform wide-area survey with angular resolution sufficient to identify HC HII regions and to match that of current infrared surveys. With a uniquely fast survey speed at $14 \mathrm{GHz}$ MeerKAT is the only instrument that is capable of such a survey. By combining a large number of small aperture dishes with broadband feeds MeerKAT has a $14 \mathrm{GHz}$ survey speed over 3 times faster than the EVLA (using the EVLA $A_{\mathrm{e}} / T_{\text {sys }}$ values given in SKA Memo \#40) and for a $14 \mathrm{GHz}$ survey requires only a similar number of pointings to an EVLA $5 \mathrm{GHz}$ survey (25). By working at high frequencies we gain an additional factor for positive spectral index sources: a MeerKAT $14 \mathrm{GHz}$ survey for HC HII regions is over 14 times faster than an EVLA $5 \mathrm{GHz}$ survey would be. With this survey speed it is possible to carry out such a survey multiple times over a baseline of a few years, finally opening the window on the variable, optically thick high frequency Galaxy.

In this paper we describe the MeerKAT Large Project MeerGAL, which will investigate the high frequency Galactic (and extragalactic) population by making first sensitive (sub-mJy) subarcsecond resolution 12-14 GHz multi-epoch survey of the Galactic Plane. MeerGAL was awarded 3300 hours of observing time on MeerKAT and will commence with the commissioning of the Xband receiver system on MeerKAT. The primary aim of MeerGAL is to discover and characterise the population of steep positive spectrum objects in the Galaxy and, for the first time, identify the variable population. However, by carefully design of MeerGAL to take full advantage of the flexible MeerKAT correlator a deep maser and recombination line survey can be simultaneously accomplished. MeerGAL will achieve the following major science goals: $i$ ) a complete and unbiased survey for young HII regions (compact, ultracompact \& hypercompact), allowing their numbers, relationship to $12.2 \mathrm{GHz}$ methanol masers, physical properties \& statistical lifetimes to be determined; ii) determine accurate mass loss rates for hundreds of OB stars as a function of their stellar parameters and search for colliding-wind binaries and flare stars; iii) measure the variability of thermal emission in the Galaxy, particularly hypercompact HII regions and planetary nebulae; iv) map the spiral structure of the Southern Galaxy by measuring radial velocities to a large sample of young HII regions and providing a complete sample of $12.2 \mathrm{GHz} \mathrm{CH}_{3} \mathrm{OH}$ masers and their phase calibrators for later VLBI parallax measurements; and $v$ ) enable the study of anomalous microwave emission and spinning dust in a wide variety of environments at high angular resolution.

In this paper we describe the scientific rationale behind MeerGAL, discuss the technical requirements of the survey, and look forward to the synergies between MeerGAL, other contemporary Galactic Plane surveys and eventually the SKA.

\section{Potential science highlights from MeerGAL}

In this section we discuss the high profile science that will result from MeerGAL, dwelling particularly on the birth and evolution of HII regions, massive stellar evolution and Galactic structure. Whilst MeerGAL was designed to address these science areas there is also significant scope for new and unexpected discoveries (26) due to the fact that MeerGAL explores genuinely new parameter 
space with sub-mJy sensitivity over sub-arcsecond angular resolution and at multiple epochs. In particular, MeerGAL has high legacy value as the highest frequency radio component of a family of Galactic Plane surveys stretching from the optical to $1.4 \mathrm{GHz}$, allowing ready cross-correlation with optical/infrared/low frequency counterparts.

\subsection{The birth and evolution of HII regions}

HII regions, and particularly the youngest and most compact HII regions, offer a unique window to peer into the early development of massive stars (27). Despite the importance of massive stars to phenomena on Galactic and extragalactic scales, many details of their early lives are still not well understood, e.g. their accretion timescales \& histories. HII regions reflect the interaction between UV radiation from the nascent massive star and their environment, and the most dense and compact HII regions (hypercompact, or HC HII regions) allow us to study single massive YSOs that have just begun to ionise their surroundings (9).

As massive stars reach the main sequence while still accreting material, this is a key phase in their development. The development of an HII region reflects the point at which the star has "switched on" its UV illumination. However it remains unclear precisely when this may occur due to the large accretion rates required to form massive stars (28), the gravitational instability and hence variability of the accretion flows $(27 ; 29)$, and potentially accretion-bloated cool massive YSOs $(30 ; 31)$. There are a number of possibilities that have been investigated theoretically. In the (32) gravitationally trapped model of HII regions, massive stars are built up by accretion onto YSOs through gravitationally trapped hypercompact HII regions surrounding the star. The (28) and (27) models both envisage HC HII regions expanding into outflow-driven cavities away from the main accretion flows. But in the (30) model, the high accretion rates (up to $\sim 10^{-3} \mathrm{M}_{\odot} \mathrm{yr}^{-1}$ ) cause the outer layers of the YSO to swell, shifting the wavelength of the peak emission into the infrared and delaying the development of an HII region until the end of the accretion phase. The duration of this effect is a function of stellar mass, YSOs more massive than $30 \mathrm{M}_{\odot}$ rapidly evolve through the bloated stage and almost immediately reach the main sequence.

All of the models above make direct predictions of the relative frequency of hypercompact HII regions: in the Keto, Peters et al., and McKee \& Tan models HC HII regions are expected to develop early and would thus be relatively common; whereas in the Hosokawa model HC HII regions would be rare, and moreover, completely absent for the highest luminosity YSOs. Unfortunately, due to the observational biases discussed in the previous section, the current observational constraints are few and far between and do not rule out any of the models. $(33 ; 19)$ find $\sim 15 \%$ of the HII regions in W49A \& Sgr B2 to be hypercompact, but these observations have limited statistics (only $\sim 8$ HC HIIs in total) and, because they are targeted toward regions of vigorous star formation, may be subject to Milky Way (i.e. "cosmic") variance. (34) found a $43 \%$ ratio of steep to flat spectrum sources in their survey of $6.7 \mathrm{GHz} \mathrm{CH} \mathrm{CH}_{3} \mathrm{OH}$ masers, but again this result is limited by small samples. For a much larger sample of UC HII regions selected from CORNISH (35) show that the most luminous YSOs are indeed associated with larger compact HII regions, which is consistent with the Hosokawa models. However, their sample is selected at $5 \mathrm{GHz}$, and can only detect the nearest HC HII regions (see Fig. 1).

To understand the birth of HII regions we need to assemble a statistically significant sample of HC HII regions. Previous surveys for young HII regions have been limited in two ways. Firstly, as 


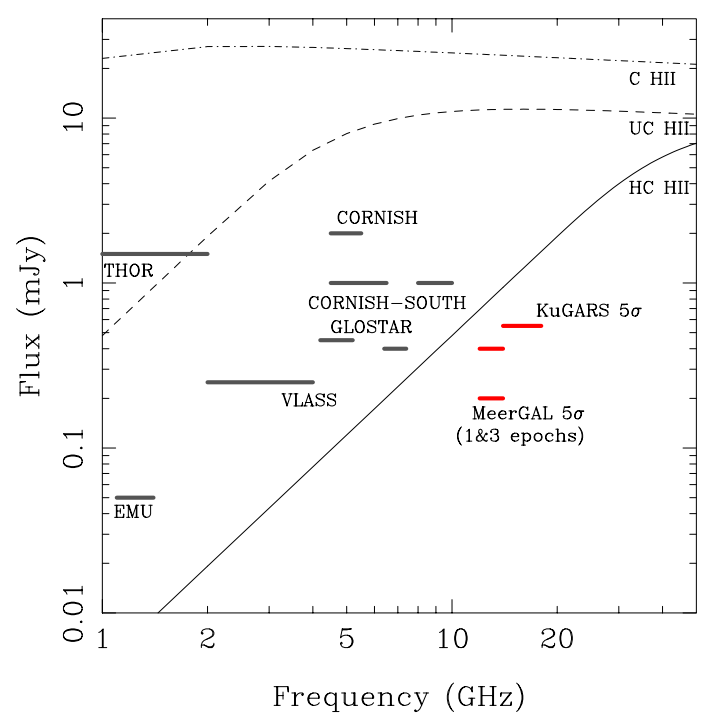

Figure 1: Theoretical spectra of homogenous isothermal HII regions at a distance of $20 \mathrm{kpc}$. Solid, dashed and dot-dashed lines show spectra for hypercompact, ultracompact and compact HII regions respectively (with diameters $0.005,0.05 \& 0.5 \mathrm{pc}$ and typical emission measures as given in Kurtz 2005). $5 \sigma$ continuum sensitivities for MeerGAL (both for 1 and after 3 epochs), KuGARS, CORNISH, GLOSTAR (5 \& 8 GHz bands) and VLASS-Galactic are indicated.

mentioned earlier, $5 \mathrm{GHz}$ surveys for young HII regions suffered a selection bias against HC HIIs (Kurtz 2005 \& see Fig. 1). Secondly, the poor $u v$ coverage of most snapshot surveys meant that they were relatively insensitive to the more extended compact HII regions (with diameters $>10^{\prime \prime}$ ). MeerGAL will avoid both of these limitations to provide a complete census of young HII regions. Our deep $14 \mathrm{GHz}$ survey ( $\sim 0.3 \mathrm{mJy} 5 \sigma$ in one epoch) ensures detections of both flat spectrum optically thin and steep spectrum optically thick HII regions to the edge of the Milky Way (see Fig. 1). The single array configuration and excellent $u v$ coverage of MeerKAT also means that we can select appropriate $u v$ tapers for different sized HII regions.

In the MeerGAL survey field (described further in Sect. 3) we expect to detect on the order of a thousand compact HII regions and a comparable number of UC HII regions, based on scaling from the area of the CORNISH $5 \mathrm{GHz}$ survey (6) and other studies (36). We estimate that we will detect somewhere between 100-500 HC HIIs in MeerGAL, taking a lower limit from estimates of their lifetime compared to UC HIIs and an upper limit from the fraction of X-band detections of a limited sample of High Mass Protostellar Objects (37). We note that one of the prime tasks of MeerGAL is to determine precise numbers and hence statistical lifetimes for these regions, testing models of HII region formation such as gravitational trapping (32). We will increase the handful of known HC HII regions $(38 ; 9 ; 39)$ by at least an order of magnitude, giving us an exclusive view into the short transition phase where rapid accretion onto the massive central protostar ceases and a detectable HII region is formed (39). Determining the point at which accretion ceases (or even if the accretion is stochastic rather than smooth) is crucial to understanding how massive stars form and how the formation process gives rise to the spectrum and distribution of stars observed in massive stellar clusters (27). 
MeerGAL will revolutionise the study of young HII regions by providing a complete and statistically significant sample of all classes of young HII regions. This allows trends in the sample to be investigated across the Galaxy, including the statistical lifetimes of the hypercompact phase for the first time which will discriminate between accretion models of massive star formation. The multiple survey epochs of MeerGAL will also pioneer the study of variability in a large sample of young HII regions, enabling us to measure the expansion rates of the nearest (i.e. within $\sim$ a few kpc) UC HII regions via difference imaging (40) and the possibility of stochastic accretion and/or bulk ordered motions in HC HII regions $(17 ; 27)$. In addition, we will also obtain simultaneous 12.2 $\mathrm{GHz} \mathrm{CH}_{3} \mathrm{OH}$ maser and hydrogen Radio Recombination Line (RRL) observations, confirming the close relationship between masers \& HII regions and the link between maser \& HII region variability. The detection of hydrogen RRLs (and also He \& CO RRLs toward bright objects) will also enable the derivation of electron temperatures, radial velocities, velocity dispersions (38, identifying broad-line HC HII regions,) and studies of the Galactocentric metallicity of HII regions (41) and Galactic gradients in electron temperature (42).

\subsection{Massive stars: Mass-loss, clumping and evolution}

MeerGAL will resolve current serious uncertainties in the mass loss and energy feedback processes of massive stars by conducting a deep survey of a number of well-known massive clusters. The target clusters provide powerful laboratories for stellar physics studies focusing on quantifying stellar mass loss rates and binary properties and interactions; the twin drivers of massive stellar evolution. Recent results have strongly challenged the current model of mass loss via stellar winds, with the enormous consequence that currently accepted mass-loss rates of luminous massive stars may be too high by an order-of-magnitude or more $(43 ; 44$, see). This has far reaching consequences for the evolution and fate of massive stars (which is largely determined by mass loss) and for galactic chemical evolution (where mass loss drives chemical and mechanical feedback on the interstellar medium). One of the most important issues is the significant discordance in the extent of mass loss occurring via clumped and/or porous radiation-driven winds. The mass-loss uncertainty (and wind porosity) has profound implications for broad astrophysical domains, including stellar evolution and the mass-loss process across the H-R diagram, and the nature of the energy and enriched gas injected into the ISM may be quite different.

Utilising the properties of the uniquely rich and accessible population of massive stars in clusters in the Galactic Plane, we will be able to systematically investigate the role of evolution in stellar mass loss. The population of the clusters covered by MeerGAL is rich enough that it samples stars at every stage of their evolution (including OB main sequence, RSG, YSG, WR, $\mathrm{LBV}, \mathrm{sgB}[\mathrm{e}]$ ), thus enabling us to directly probe the corresponding evolution of mass loss rate. The advantage of using free-free radio fluxes for determining mass loss for massive stars is that, unlike $\mathrm{H} \alpha$ and $\mathrm{UV}$, the emission arises at large radii in the stellar wind, where the terminal velocity will have been reached. The interpretation of the radio fluxes is more straightforward therefore and is not strongly dependent on details of the velocity law, ionization conditions, inner velocity field, or the photospheric profile. Significant progress can be made with MeerKAT point source detections, and spatial resolution of the wind is not a requisite.

We expect $\sim 300$ of the Reed catalogue of OB supergiants that lie in the survey area to be detectable (based on simple wind models), which means that MeerGAL will increase the number 
of OB stars detected in the radio by a factor of at least 5 . The greater geometric region and density squared dependence of the free-free flux makes the radio observations extremely sensitive to clumping in the wind. The radio will be directly compared to other density-squared diagnostics such as $\mathrm{H} \alpha$. We will use clumping models for the radio and $\mathrm{H} \alpha(45 ; 44)$ to arrive at a consistent set of mass loss rates and determine the relative amount of clumping as a function of velocity over a wide range of spectral types. We will investigate for the first time how clumping (or volume filling factor) changes as a function of fundamental stellar parameters (e.g. temperature, luminosity, rotation velocity), providing powerful constraints on physical models for the origins of clumping and hence reliable estimates of mass-loss rate as a function of stellar mass.

We plan that an additional science return from our survey of clusters will ultimately be to (i) provide a better determined binary star frequency in clusters, which is an important constraint for evolutionary population synthesis models, and hence having a broad impact on our understanding of galactic chemical evolution; (ii) study statistically the colliding-wind phenomenon and better understand its dependence on stellar and binary parameters. This aspect of the project will require follow-up MeerKAT data at $1.4 \mathrm{GHz}$ and $8 \mathrm{GHz}$ (via the standard proposal route), to unambiguously determine between thermal and non-thermal emission. Fundamentally, where a star is found to have non-thermal emission, a colliding wind and hence a binary companion can be inferred. This is a highly efficient way to identify binaries in clusters, as only a single-epoch observation is required. Knowing the binary frequency over the whole range is important for evolutionary population synthesis codes. In addition, the properties of massive binaries, in particular the mass ratios and separation distributions, resulting in strong constraints on star formation models (46).

We will additionally analyse the MeerKAT massive stellar cluster data for possible strong $\mathrm{H} \alpha$ emission-line objects that could be members of larger population of lower mass pre-main sequence stars that have yet to be uncovered. VLA studies at $\sim 5 \mathrm{GHz}$ have shown that $\sim 10 \%$ of Weak Line $\mathrm{T}$ Tauris (WTTS) show radio emission at levels of $10^{16}-10^{17} \mathrm{erg} \mathrm{s}^{-1} \mathrm{~Hz}^{-1}$ (47) and the majority of WTTS at levels of approximately $10^{15} \mathrm{erg} \mathrm{s}^{-1} \mathrm{~Hz}^{-1}$ (48), which is a hundred times more intense than the Sun, indicating that magnetic activity is much stronger during the PMS phase than in later phases of their evolution. The stronger emitters, and those belonging to relative close clusters, will be detectable in the observations proposed here. Flaring during the observations will make even more stars detectable.

What is required in order to disentangle the effects of age, stellar mass, and environment are not just large samples, but specifically samples over a range in stellar mass in high-concentration star-forming regions where the potential environmental effect of the OB stars is available. Several of the clusters in the MeerGAL survey field are suitable for this task. For individual objects, MeerGAL enables us to compare the radio properties with the $\mathrm{H} \alpha$ emission in relation to the PMS spectral type and rotational velocities, which are believed to play a fundamental role in the production of non-thermal radio emission of WTTS. On the larger scale, the data will allow us to investigate the nature of the ongoing star formation in stellar clusters and study potential triggered star formation scenarios (49).

\subsection{The structure of the Southern Milky Way}

In parallel with its deep continuum survey MeerGAL will also carry out a shallower line survey of hydrogen radio recombination lines (RRLs) which will enable the construction of an 
unprecedented 3-dimensional map of the Southern Galactic Plane and its massive star forming regions. The current "standard model" for the structure of the Milky Way is that of (50), hereafter referred to as GG76 - see Fig. 2. This study was originally based on the RRL radial velocity and spectrophotometric distance measurements of 268 Giant HII regions, later refined to include pulsar dispersion measures $(51 ; 52 ; 53)$. Despite being subject to significant uncertainty from kinematic distances the GG76 model of 4 major spiral arms has remained the basis of our understanding of the Milky Way's structure for over 30 years and forms the template for a number of more recent studies $(54 ; 55)$.
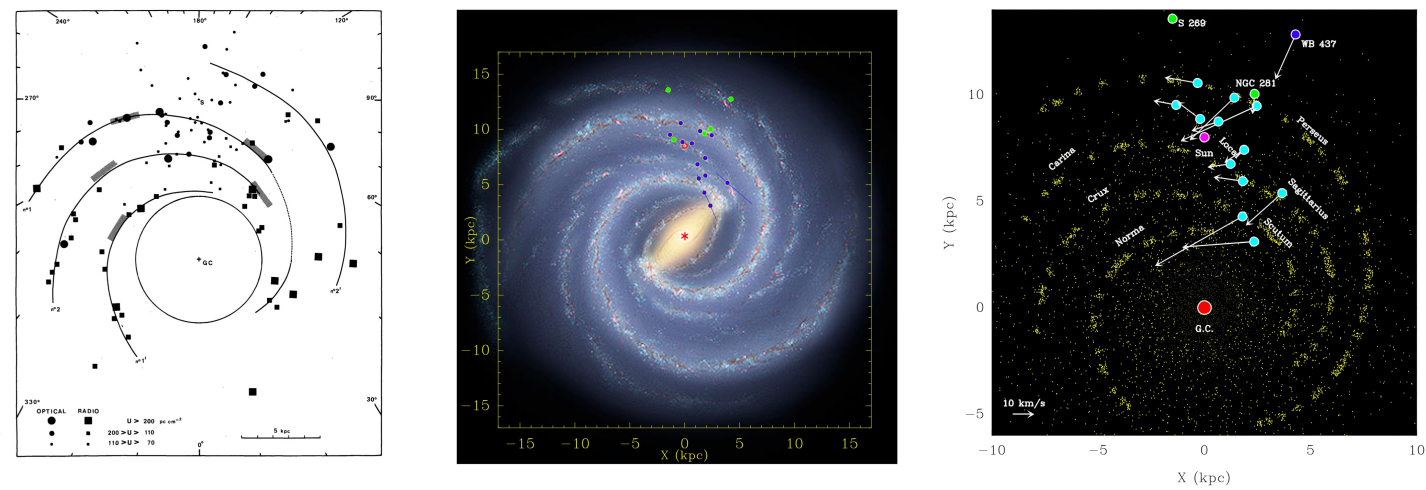

Figure 2: Left: The Georgelin \& Georgelin (1976) model, based on HII region recombination line velocities and stellar luminosity distances. Middle: An artists impression of the Milky Way, created from the 2-

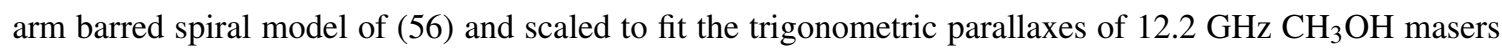
(57). Right: The (51) refinement of the Georgelin \& Georgelin model with overlaid positions and peculiar velocities of $\mathrm{H}_{2} \mathrm{O}$ masers observed with VERA and $12.2 \mathrm{GHz} \mathrm{CH}_{3} \mathrm{OH}$ masers observed with the VLBA. Note the complete absence of any trigonometric maser parallaxes in the Southern Galactic Plane (i.e. the Norma, Crux \& Carina arms).

However, significant disagreement has emerged in both the number and placement of spiral arms in the GG76 model, and in the overall mass and rotation curve of the Milky Way. Different models suggest as few as 2 spiral arms and as many as 6 spiral arms (58). Recent VLBI parallax measurements of $12.2 \mathrm{GHz} \mathrm{CH} \mathrm{CH}_{3} \mathrm{OH}$ masers suggest that the Milky Way rotation speed is $\sim 15 \%$ faster than previously assumed, either potentially increasing the Milky Way mass by $\sim 50 \%(57 ; 59)$ or the Sun's circular velocity (60). An accurate map of the Milky Way's spiral structure, rotation curve and basic properties is vital to a number of widely different fields, for example in determining luminosities and masses of star forming regions (35); relating the local Milky Way Tully-Fisher relation to external galaxies (is the Milky Way underluminous for its mass? - e.g. (61); and understanding the Milky Way's dark matter distribution, which is particularly relevant to predictions for Dark Matter direct detection experiments (62).

MeerGAL will provide a 21st century revision of the GG76 model for the Carina, Crux \& Norma spiral arms, providing a backdrop of RRL and $12.2 \mathrm{GHz}$ maser radial velocity measurements against which spectrophotometry and VLBI maser parallaxes can accurately calibrate the resulting rotation curve. At the sensitivity levels required to detect HC HII regions and stellar winds we estimate that we will be able to measure RRL radial velocities for several hundred objects with accuracy of a few $\mathrm{km} \mathrm{s}^{-1}$ using a spectral stacking analysis to enhance sensitivity to 
faint lines (7, e.g. as used in THOR,). We describe the feasibility of this technique in Sect. 3, but we note in passing here that 40 years of hardware development has reached the point at which the 32k channel "continuum" mode of MeerKAT has the same spectral resolution as the single-dish spectral line receivers used to measure the hydrogen RRLs for the GG76 model. To ensure uniform sampling in longitude velocity we also plan to conduct deeper observations of selected HII regions (requiring only 2 hours per HII region). To this total we add radial velocities from $\sim 100012.2 \mathrm{GHz}$ masers detected in parallel with the survey observations, which means that we will be able to place many more points on our derived rotation curve than in the entire GG76 model. These accurately positioned masers and nearby background radio galaxies (i.e. phase calibrators) will be a valuable resource for future VLBI studies (for example with the African VLBI Network).

Kinematic distances derived for compact and ultracompact HII regions offer significant advantages over the diffuse HII regions used in the GG76 model. Their smaller size allows a more accurate position to be determined on the sky and they are readily observable in large numbers. The well-known kinematic distance ambiguity, caused by multiple solutions to the rotation curve for inner Galaxy objects, is much easier to break for compact \& ultracompact HII regions (63). HI data from the existing SGPS HI survey and forthcoming ASKAP surveys will be used to solve the distance ambiguity for our HII region sample $(63 ; 64)$. Finally, there is a much closer relationship between the $12.2 \mathrm{GHz}$ masers used in VLBI parallax measurements to young compact \& ultracompact HII regions than diffuse HII regions. We will be able to tie together VLBI parallax distances and RRL kinematic distances to single star forming complexes, enabling a common"distance ladder" for the Carina, Crux \& Norma Arms.

\section{Survey Design \& Observational Strategy}

MeerGAL is carefully designed in order to maximise observing efficiency while effectively meeting the major science goals outlined in the previous section. The major drivers of the MeerGAL design are outlined below.

\subsection{Survey Area}

The MeerGAL survey area covers the GLIMPSE-S survey field, with a longitude extension to include the tangent point of the Carina Arm, i.e. $280^{\circ} \leq l \leq 350^{\circ}$ and $|b| \leq 1^{\circ}$ (see Fig. 3, right panel). This area maximises the number of compact, ultracompact and hypercompact regions that we expect to detect (Fig 3, left panel), in addition to covering a number of promininent massive stellar clusters and the full extent of the Carina, Crux \& Norma Arms. Our strategy to map the MeerGAL survey area follows that of the successful CORNISH $5 \mathrm{GHz}$ galactic plane survey, of which a number of the MeerGAL team were involved. We will map the survey area using the NVSS hexagonal pointing pattern (65) scaled to the MeerKAT primary beam at $14 \mathrm{GHz}$ (i.e. with spacing $\theta_{\text {hex }}=0.09^{\circ}$ compared to the primary beam of $0.12^{\circ}$ ). To cover the total survey area of 140 square degrees requires 20000 pointings, comparable to the number of pointings carried out by CORNISH. To improve $u v$ coverage of each pointing at least two widely spaced repeats per epoch will be observed, a strategy that worked well for CORNISH (6). 

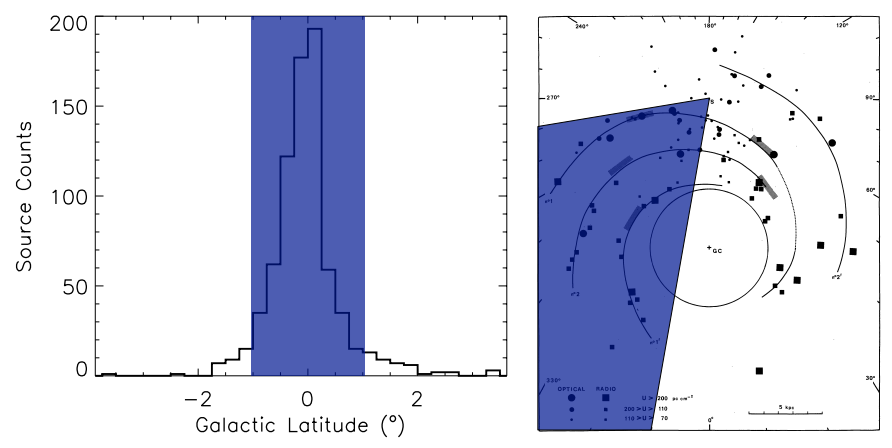

Figure 3: Left: The latitude distribution of UC HII regions (66), showing that most massive star forming regions are found within $1^{\circ}$ of the mid-plane due to their small scale-height. Right: The spiral arm model of (50). The proposed survey area is indicated by blue shading.

\subsection{Depth}

The required depth of MeerGAL is ultimately driven by the need to measure the spectral index of hypercompact HII regions and massive stellar winds. In order to be able to simultaneously detect line and continuum emission we will use the $32 \mathrm{k}$ channel mode of the correlator, binning channels to achieve the desired continuum sensitivity. This approach will also minimise bandwidth smearing. The spectral resolution of this correlator mode is adequate for the $\sim 20 \mathrm{~km} \mathrm{~s}^{-1}$ wide RRLs and for maser detection experiments.

The required continuum sensitivity in a single epoch is estimated from the flux of a small isothermal homogenous HC HII region at the far edge of the Galaxy $\left(d \sim 20 \mathrm{kpc}, T_{\mathrm{e}} \sim 10^{4} \mathrm{~K}\right.$, $r \sim 1000 \mathrm{AU}$ ), which has a $14 \mathrm{GHz}$ flux of $0.9 \mathrm{mJy}$, falling to $0.7 \mathrm{mJy}$ at $12 \mathrm{GHz}$. The estimated integration time required to detect such an object is determined using the equations in J. Cordes' SKA Survey Memo \#109. Assuming a continuum bandwidth of $400 \mathrm{MHz}$, an $A_{\mathrm{e}} / T_{\text {sys }}$ value of 200 , a tapering factor of 1.9 (to reach a $0.8^{\prime \prime}$ FWHM beam), a factor 1.5 to account for $T_{\text {sys }}$ and Ruze losses at $14 \mathrm{GHz}$ (J. Jonas, priv comm) and averaging both polarisations we obtain a $5 \sigma$ detection in 120s of integration with an rms noise of $130 \mu \mathrm{Jy} / \mathrm{beam}$. Combined over the full band of $\sim 1$ GHz this sensitivity amounts to $\sim 80 \mu \mathrm{Jy} /$ beam and over the full 3 epoch survey we expect to reach a $1 \sigma$ sensitivity of $\sim 40 \mu \mathrm{Jy} /$ beam (assuming a $2 \mathrm{GHz}$ bandwidth in epoch 3 ). The resolution of MeerKAT at $14 \mathrm{GHz}\left(0.8^{\prime \prime}\right)$ is well matched to the diameters of $\mathrm{HC}$ and UC HII regions (though UC/HC HII regions will not likely be resolved beyond $\sim 10 \mathrm{kpc}$, this does not affect our science goals). More extended HII regions can be identified ( $\sim 10$ s of arcsec) by tapering our data to a resolution of $4^{\prime \prime}$ or greater. The high dynamic range of MeerKAT will achieve this depth over all regions except for those near sources of $\sim 10 \mathrm{Jy}$ or brighter. CORNISH shows that sources this bright are relatively rare (25).

At the same time RRL data will be obtained by binning the spectrum into $5 \mathrm{~km} \mathrm{~s}^{-1}$ channels. One MeerGAL epoch will result in an rms noise of $7.3 \mathrm{mJy} /$ beam per $5 \mathrm{~km} \mathrm{~s}^{-1}$ channel. This is more than sufficient to detect masing lines, such as the $12.2 \mathrm{GHz} \mathrm{CH}_{3} \mathrm{OH}$ maser. For RRLs, at least another factor of 2 will be gained in sensitivity by stacking the $4 \alpha$-lines falling in the bandpass (and eventually a factor of 3 in epoch 3 ). For HII regions that are not initially detected in the binned 
spectrum, an iterative stacking analysis will be used to extract potential RRL detections based on the well known frequency separations of the RRLs. Finally, to enhance the RRL sensitivity a taper can be applied to reach a 4 " synthesised beam and select sensitivity over angular resolution.

After combining 3 epochs the resulting $5 \sigma$ detection threshold for stacked RRLs will be $\sim 6$ mJy. For a typical line-to-continuum ratio of $15 \%$ for $14 \mathrm{GHz}$ RRLs this equates to detecting recombination line emission toward all thermal sources brighter than $40 \mathrm{mJy}$. We estimate the fraction of sources from the source counts as a function of flux from the CORNISH 5 GH survey. Depending on the spectral index of the source MeerGAL will also gain a $\sim 1.5$ factor in sensitivity over CORNISH (UC HII regions still have a rising spectrum at $14 \mathrm{GHz}$, see Fig. 1). In total of the order of a few hundred sources are expected to be detected in recombination lines, scaling from the CORNISH $5 \mathrm{GHz}$ source counts. So that we can maintain a uniform longitude-velocity coverage for our RRL sample it is also planned for deeper MeerKAT follow-up of a selection of fainter HII regions with undetected RRLs in the main survey. Around 100 hours per epoch will be spent on this follow-up.

\subsection{Cadence}

The cadence of the survey is driven by the timescale for variability of HC HII regions, masers and planetary nebulae. These objects have been shown to vary over typical timescales of a year or longer $(17 ; 67 ; 20)$, although short-period month-long variations have been observed toward some 12.2 GH masers (68). Year to year variations for the variable HC HII regions that has been discovered are $\sim 10 \%$ per year, which is well within the capability of MeerGAL to measure. MeerGAL is thus split into yearly epochs spanning a two year baseline with three independent measurements of the flux of each object. Each epoch will consist of a complete pass over the survey area. These three survey epochs allow us to gradually build up the sensitivity of MeerGAL, while maintaining the capability to measure variable emission and also fully exploiting the phased increase in MeerKAT capability.

\section{Synergies with contemporary surveys and looking forward to the SKA}

MeerGAL is highly complementary to a number of currently ongoing or planned surveys of the Galactic Plane, slotting neatly into the comprehensive and currently growing family of high angular resolution, high sensitivity surveys. From Table 1 it can be seen that following completion of MeerGAL the Southern Galactic Plane will be covered in a multitude of wavelengths from the optical, through far-infrared to long wavelength radio. To the list of surveys in Table 1 we may also add a number of relevant all-sky surveys, which have covered or will cover the Galactic Plane: the VISTA Hemisphere Survey (VHS) in the near-IR; WISE, spanning 3.4-22 $\mu \mathrm{m}$; the Akari mid and far-IR survey; the Planck Surveyor CMB LFI \& HFI surveys; and the ASKAP survey EMU which will make the deepest $1.4 \mathrm{GHz}$ survey of the southern sky yet attempted $(69,10 \mu \mathrm{Jy}, 1 \sigma$;).

MeerGAL will occupy a central place in this family of surveys as the deepest survey for thermal radio emission in the Galactic Plane, with an angular resolution that is ideally matched to optical and infrared surveys. In the age of the Virtual Observatory MeerGAL will be the destination of choice for comparison radio data (including variability) to the literally billions of Galactic Plane objects that are being discovered in VVV, VPHAS+, GLIMPSE360 \& WISE. The depth and 
Table 1: List of current and planned Galactic Plane surveys. Note that for reasons of space we have omitted surveys with purely Northern components.

\begin{tabular}{lllll}
\hline Name & Survey area & Wavelengths & Sensitivity & Resolution \\
\hline VPHAS+ & $210<l<35,|b|<5$ & $\mathrm{u}^{\prime} \mathrm{g}^{\prime} \mathrm{r}^{\prime} \mathrm{i}^{\prime} \mathrm{H} \alpha$ & $\mathrm{r}^{\prime}<21$ & $<1.2^{\prime \prime}$ \\
VVV & $295<l<10,|b|<2$ & ZYJHK $_{s}$ & $\mathrm{~K}_{s}<18(1$ epoch $)$ & $0.8^{\prime \prime}$ \\
GLIMPSE & $295<l<65,|b|<1$ & $3.6-8 \mu \mathrm{m}$ & $\sim 0.4 \mathrm{mJy}(1 \sigma)$ & $1^{\prime \prime}$ \\
GLIMPSE360 & $0<l<360,|b| \lesssim 1$ & $(3.6,4.5 \mu \mathrm{m})$ & $\sim 0.4 \mathrm{mJy}(1 \sigma)$ & $\sim 1^{\prime \prime}$ \\
MIPSGAL & $298<l<63,|b|<1$ & $24,70 \mu \mathrm{m}$ & $0.3,15 \mathrm{mJy}(1 \sigma)$ & $6^{\prime \prime}, 18^{\prime \prime}$ \\
Hi-GAL & $300<l<60,|b|<1$ & $70-500 \mu \mathrm{m}$ & $\sim 20-30 \mathrm{mJy}$ & $7^{\prime \prime}-36^{\prime \prime}$ \\
ATLASGAL & $300<l<60,|b|<1.5$ & $870 \mu \mathrm{m}$ & $50 \mathrm{mJy}(1 \sigma)$ & $19^{\prime \prime}$ \\
MeerGAL & $280<l<350,|b|<1$ & $2 \mathrm{~cm}$ & $60 \mu \mathrm{Jy}(1 \sigma)$ & $0.8^{\prime \prime}$ \\
CORNISH-S & $295<l<65,|b|<1$ & $6 \mathrm{~cm}$ & $0.14 \mathrm{mJy}(1 \sigma)$ & $3^{\prime \prime}$ \\
MMB & $180<l<60,|b|<2$ & $(6.7 \mathrm{GHz})$ & $0.2 \mathrm{Jy}(1 \sigma)$ & $\sim 0.1^{\prime \prime}$ \\
GASKAP & $180<l<80,|b|<2.5$ & $21 \mathrm{~cm}$ & $0.6 \mathrm{mJy}(1 \sigma)$ & $20^{\prime \prime}$ \\
\hline
\end{tabular}

common survey area of MeerGAL to that of many other survey projects will ensure wide use of its data for considerable time to come.

The wealth of readily available Galactic Plane survey data from the optical to the long-wavelength radio also has strong benefits for MeerGAL science. For example, the work on stellar wind clumping and Classical T Tauris will be greatly aided by accurate $\mathrm{H} \alpha$ and broadband optical/IR photometry from VPHAS + \& VVV. The galactic structure measured by MeerGAL can be compared to that derived independently from Cepheids \& RR Lyrae variables identified in VVV. Studies of the host molecular cloud clumps of UC/HC HII regions will benefit from well-sampled SEDs from the radio to mid-IR for almost the entire survey area. We can compare the positions of TeV/gamma ray sources from HESS/MAGIC to our radio catalogues to identify their possible MeerGAL counterparts. In short, MeerGAL is ideally placed to take full advantage of our forthcoming panoramic multi-wavelength view of the Galaxy.

Although we have dwelt mainly upon synergies with Southern Galactic Plane surveys it is worth mentioning the Northern Hemisphere sister project to MeerGAL, KuGARS: the Ku-band Galactic Reconnaissance Survey (PI Thompson). KuGARS is a proposed JVLA 14-18 GHz survey of the Northern Galactic Plane between $10^{\circ} \leq l \leq 65^{\circ}$ and $|b| \leq 1$. The depth, angular resolution and goals of KuGARS are similar to MeerGAL (see Fig. 1) but cover only one epoch of observations and so KuGARS cannot explore the variability of detected sources. In addition to continuum science KuGARS also includes zoom windows on important spectral lines in the $4 \mathrm{GHz}$ bandpass (the $\mathrm{CH}_{3} \mathrm{OH}$ maser at $12.2 \mathrm{GHz}$, excited $\mathrm{OH}$ maser at $13.4 \mathrm{GHz}, \mathrm{H}_{2} \mathrm{CO}$ at $14.4 \mathrm{GHz}$ and $5 \mathrm{RRLs}$ ) with the aim of measuring Zeeman splitting in $\mathrm{OH}$ and $\mathrm{CH}_{3} \mathrm{OH}$ masers and investigating $\mathrm{H}_{2} \mathrm{CO}$ absorption towards continuum sources. KuGARS has completed pilot observations of a $4 \times 2^{\circ}$ region around W49A and will shortly submit a proposal to JVLA to cover the remaining survey region.

Finally, MeerGAL will play an important role in planning future projects with the SKA. MeerGAL will provide a shallow initial tier at SKA band 5 frequencies that can be used to plan further deeper continuum surveys such as those proposed by (70). QSOs discovered in MeerGAL will be 
important position calibrators for SKA VLBI parallax observations of Galactic masers (71, see). The Radio Recombination Lines discovered in MeerGAL will set the scene for wide-area broadband RRL surveys (72) that will link the small angular scales probed by MeerGAL to the wider scales traced by lower frequency lines with the SKA. And, with a small frequency adjustment to incorporate the $14.4 \mathrm{GHz} \mathrm{H_{2 }} \mathrm{CO}$ line MeerGAL could also be used to investigate absorption against Galactic continuum sources in preparation for full-scale anomalous absorption studies with the SKA against the CMB (72).

\section{References}

[1] M. S. Longair, Unveiling the distant universe, Astronomy and Geophysics 39 (Apr., 1998) 10.

[2] J. J. Condon, Very Large Radio Surveys of the Sky, Proceedings of the National Academy of Science 96 (Apr., 1999) 4756-4758.

[3] A. J. Green, L. E. Cram, M. I. Large and T. Ye, The Molonglo Galactic Plane Survey. I. Overview and Images, ApJS 122 (May, 1999) 207-219, [astro-ph/9810385].

[4] D. J. Helfand, R. H. Becker, R. L. White, A. Fallon and S. Tuttle, MAGPIS: A Multi-Array Galactic Plane Imaging Survey, AJ 131 (May, 2006) 2525-2537.

[5] A. R. Taylor, S. J. Gibson, M. Peracaula, P. G. Martin, T. L. Landecker, C. M. Brunt et al., The Canadian Galactic Plane Survey, AJ 125 (June, 2003) 3145-3164.

[6] M. G. Hoare, C. R. Purcell, E. B. Churchwell, P. Diamond, W. D. Cotton, C. J. Chandler et al., The Coordinated Radio and Infrared Survey for High-Mass Star Formation (The CORNISH Survey). I. Survey Design, PASP 124 (Sept., 2012) 939-955, [1208.3351].

[7] S. Bihr, K. G. Johnston, H. Beuther, L. D. Anderson, J. Ott, M. Rugel et al., Continuum sources from the THOR survey between 1 and 2 GHz, A\&A 588 (Apr., 2016) A97, [1601.03427].

[8] M. Lacy, S. A. Baum, C. J. Chandler, S. Chatterjee, E. J. Murphy, S. T. Myers et al., The VLA Sky Survey (VLASS): Description and Science Goals, in American Astronomical Society Meeting Abstracts, vol. 227 of American Astronomical Society Meeting Abstracts, p. 324.09, Jan., 2016.

[9] S. Kurtz, Hypercompact HII regions, in Massive Star Birth: A Crossroads of Astrophysics (R. Cesaroni, M. Felli, E. Churchwell and M. Walmsley, eds.), vol. 227 of IAU Symposium, pp. 111-119, 2005, DOI.

[10] P. G. Mezger and A. P. Henderson, Galactic H II Regions. I. Observations of Their Continuum Radiation at the Frequency $5 \mathrm{GHz}$, ApJ 147 (Feb., 1967) 471.

[11] G. Anglada, Radio Jets in Young Stellar Objects, in Radio Emission from the Stars and the Sun (A. R. Taylor and J. M. Paredes, eds.), vol. 93 of Astronomical Society of the Pacific Conference Series, pp. 3-14, 1996.

[12] I. Bains, M. Cohen, J. M. Chapman, R. M. Deacon and M. P. Redman, Revealing the transition from post-AGB stars to planetary nebulae: non-thermal and thermal radio continuum observations, MNRAS 397 (Aug., 2009) 1386-1401.

[13] T. Handa, Y. Sofue, N. Nakai, H. Hirabayashi and M. Inoue, A radio continuum survey of the Galactic plane at $10 \mathrm{GHz}$, PASJ 39 (1987) 709-753.

[14] G. Langston, A. Minter, L. D'Addario, K. Eberhardt, K. Koski and J. Zuber, The First Galactic Plane Survey at 8.35 and 14.35 GHZ, AJ 119 (June, 2000) 2801-2827. 
[15] T. Murphy, E. M. Sadler, R. D. Ekers, M. Massardi, P. J. Hancock, E. Mahony et al., The Australia Telescope $20 \mathrm{GHz}$ Survey: the source catalogue, MNRAS 402 (Mar., 2010) 2403-2423, [0911.0002].

[16] J. M. Cordes, T. J. W. Lazio and M. A. McLaughlin, The dynamic radio sky, NAR 48 (Dec., 2004) 1459-1472, [astro-ph/0410045].

[17] R. Galván-Madrid, L. F. Rodríguez, P. T. P. Ho and E. Keto, Time Variation in G24.78+0.08 A1: Evidence for an Accreting Hypercompact H II Region?, ApJL 674 (Feb., 2008) L33, [0801.1298].

[18] M. Klassen, T. Peters and R. E. Pudritz, H II Region Variability and Pre-main-sequence Evolution, ApJ 758 (Oct., 2012) 137, [1208.6001].

[19] C. G. De Pree, T. Peters, M.-M. Mac Low, D. J. Wilner, W. M. Goss, R. Galván-Madrid et al., Flickering of $1.3 \mathrm{~cm}$ Sources in Sgr B2: Toward a Solution to the Ultracompact H II Region Lifetime Problem, ApJL 781 (Feb., 2014) L36.

[20] A. A. Zijlstra, P. A. M. van Hoof and R. A. Perley, The Evolution of NGC 7027 at Radio Frequencies: A New Determination of the Distance and Core Mass, ApJ 681 (July, 2008) 1296-1309, [0 801.3327$]$.

[21] S. D. Hyman, T. J. W. Lazio, N. E. Kassim, P. S. Ray, C. B. Markwardt and F. Yusef-Zadeh, A powerful bursting radio source towards the Galactic Centre, Nature 434 (Mar., 2005) 50-52, [astro-ph/0503052].

[22] D. P. Finkbeiner, D. J. Schlegel, C. Frank and C. Heiles, Tentative Detection of Electric Dipole Emission from Rapidly Rotating Dust Grains, ApJ 566 (Feb., 2002) 898-904, [astro-ph/0109534].

[23] S. Casassus, C. Dickinson, K. Cleary, R. Paladini, M. Etxaluze, T. Lim et al., Centimetre-wave continuum radiation from the $\rho$ Ophiuchi molecular cloud, MNRAS 391 (Dec., 2008) 1075-1090, [0 809.3965$].$

[24] Planck Collaboration, P. A. R. Ade, N. Aghanim, M. Arnaud, M. Ashdown, J. Aumont et al., Planck early results. XX. New light on anomalous microwave emission from spinning dust grains, A\&A 536 (Dec., 2011) A20, [1101.2031].

[25] C. R. Purcell, M. G. Hoare, W. D. Cotton, S. L. Lumsden, J. S. Urquhart, C. Chandler et al., The Coordinated Radio and Infrared Survey for High-mass Star Formation. II. Source Catalog, ApJS 205 (Mar., 2013) 1, [1211. 7116].

[26] P. Wilkinson, The SKA and the Unknown Unknowns, Advancing Astrophysics with the Square Kilometre Array (AASKA14) (Apr., 2015) 65, [1506.08540].

[27] T. Peters, R. Banerjee, R. S. Klessen, M.-M. Mac Low, R. Galván-Madrid and E. R. Keto, H II Regions: Witnesses to Massive Star Formation, ApJ 711 (Mar., 2010) 1017-1028, [1 001.2470$]$.

[28] C. F. McKee and J. C. Tan, The Formation of Massive Stars from Turbulent Cores, ApJ 585 (Mar., 2003) 850-871, [astro-ph/0206037].

[29] R. Kuiper and H. W. Yorke, On the Simultaneous Evolution of Massive Protostars and Their Host Cores, ApJ 772 (July, 2013) 61, [1305.6310].

[30] T. Hosokawa and K. Omukai, Low-Metallicity Protostars and the Maximum Stellar Mass Resulting from Radiative Feedback: Spherically Symmetric Calculations, ApJ 703 (Oct., 2009) 1810-1818, [0906.1607].

[31] T. Hosokawa, H. W. Yorke and K. Omukai, Evolution of Massive Protostars Via Disk Accretion, ApJ 721 (Sept., 2010) 478-492, [1 005 . 2827]. 
[32] E. Keto, The Formation of Massive Stars by Accretion through Trapped Hypercompact H II Regions, ApJ 599 (Dec., 2003) 1196-1206, [astro-ph/ 0309131$].$

[33] C. G. De Pree, D. M. Mehringer and W. M. Goss, Multifrequency, High-Resolution Radio Recombination Line Observations of the Massive Star-forming Region W49A, ApJ 482 (June, 1997) 307-333.

[34] Á. Sánchez-Monge, M. T. Beltrán, R. Cesaroni, F. Fontani, J. Brand, S. Molinari et al., Different evolutionary stages in massive star formation. Centimeter continuum and $\mathrm{H}_{2} \mathrm{O}$ maser emission with ATCA, A\&A 550 (Feb., 2013) A21, [1211.0847].

[35] J. S. Urquhart, T. J. T. Moore, T. Csengeri, F. Wyrowski, F. Schuller, M. G. Hoare et al., ATLASGAL - towards a complete sample of massive star forming clumps, MNRAS $\mathbf{4 4 3}$ (Sept., 2014) 1555-1586, [1406.5078].

[36] T. M. Bania, L. D. Anderson, D. S. Balser and R. T. Rood, The Green Bank Telescope Galactic H II Region Discovery Survey, ApJL 718 (Aug., 2010) L106-L111, [1 006.5929 ].

[37] T. K. Sridharan, H. Beuther, P. Schilke, K. M. Menten and F. Wyrowski, High-Mass Protostellar Candidates. I. The Sample and Initial Results, ApJ 566 (Feb., 2002) 931-944, [astro-ph/0110363].

[38] M. Sewilo, E. Churchwell, S. Kurtz, W. M. Goss and P. Hofner, Broad Radio Recombination Lines from Hypercompact H II Regions, ApJ 605 (Apr., 2004) 285-299.

[39] M. Sewiło, E. Churchwell, S. Kurtz, W. M. Goss and P. Hofner, Internal Dynamics of the Hypercompact H II Region G28.20-0.04N, ApJ 681 (July, 2008) 350-354, [0 803.2872 ].

[40] J. M. Acord, E. Churchwell and D. O. S. Wood, The Expansion Rate of and Distance to G5.89-0.39, ApJL 495 (Mar., 1998) L107-L110.

[41] A. Afflerbach, E. Churchwell, J. M. Acord, P. Hofner, S. Kurtz and C. G. Depree, Galactic Temperature and Metallicity Gradients from Ultracompact H II Regions, ApJS 106 (Oct., 1996) 423.

[42] M. I. R. Alves, M. Calabretta, R. D. Davies, C. Dickinson, L. Staveley-Smith, R. J. Davis et al., The HIPASS survey of the Galactic plane in radio recombination lines, MNRAS 450 (June, 2015) 2025-2042, [1411.4497].

[43] A. W. Fullerton, D. L. Massa and R. K. Prinja, The Discordance of Mass-Loss Estimates for Galactic O-Type Stars, ApJ 637 (Feb., 2006) 1025-1039, [astro-ph/ 0510252 ].

[44] J. Puls, N. Markova, S. Scuderi, C. Stanghellini, O. G. Taranova, A. W. Burnley et al., Bright $O B$ stars in the Galaxy. III. Constraints on the radial stratification of the clumping factor in hot star winds from a combined H, IR and radio analysis, A\&A 454 (Aug., 2006) 625-651, [astro-ph/0604372].

[45] R. Blomme, R. K. Prinja, M. C. Runacres and S. Colley, Radio and submillimetre observations of $<A S T R O B J>$ epsilon Ori $</ A S T R O B J>$, A\&A 382 (Feb., 2002) 921-934.

[46] S. P. Goodwin, P. Kroupa, A. Goodman and A. Burkert, The Fragmentation of Cores and the Initial Binary Population, Protostars and Planets V (2007) 133-147, [astro-ph/0603233].

[47] D. O'Neal, E. D. Feigelson, R. D. Mathieu and P. C. Myers, A radio survey of weak T Tauri stars in Taurus-Auriga, AJ 100 (Nov., 1990) 1610-1617.

[48] J. A. Leous, E. D. Feigelson, P. Andre and T. Montmerle, A rich cluster of radio stars in the Rho Ophiuchi cloud cores, ApJ 379 (Oct., 1991) 683-688.

[49] J. S. Vink, J. E. Drew, D. Steeghs, N. J. Wright, E. L. Martin, B. T. Gänsicke et al., IPHAS discoveries of young stars towards Cyg OB2 and its southern periphery, MNRAS 387 (June, 2008) 308-318, [0803.2840]. 
[50] Y. M. Georgelin and Y. P. Georgelin, The spiral structure of our Galaxy determined from $H$ II regions, A\&A 49 (May, 1976) 57-79.

[51] J. H. Taylor and J. M. Cordes, Pulsar distances and the galactic distribution of free electrons, ApJ 411 (July, 1993) 674-684.

[52] J. M. Cordes and T. J. W. Lazio, NE2001.I. A New Model for the Galactic Distribution of Free Electrons and its Fluctuations, ArXiv Astrophysics e-prints (July, 2002), [astro-ph/0207156].

[53] J. M. Cordes and T. J. W. Lazio, NE2001. II. Using Radio Propagation Data to Construct a Model for the Galactic Distribution of Free Electrons, ArXiv Astrophysics e-prints (Jan., 2003), [astro-ph/0301598].

[54] E. Araya, P. Hofner, E. Churchwell and S. Kurtz, Arecibo Observations of Formaldehyde and Radio Recombination Lines toward Ultracompact H II Regions, ApJS 138 (Jan., 2002) 63-74.

[55] R. Paladini, R. D. Davies and G. De Zotti, Spatial distribution of Galactic HII regions, MNRAS 347 (Jan., 2004) 237-245, [astro-ph/ 0309350 ].

[56] R. A. Benjamin, The Spiral Structure of the Galaxy: Something Old, Something New..., in Massive Star Formation: Observations Confront Theory (H. Beuther, H. Linz and T. Henning, eds.), vol. 387 of Astronomical Society of the Pacific Conference Series, p. 375, May, 2008.

[57] M. J. Reid, K. M. Menten, X. W. Zheng, A. Brunthaler, L. Moscadelli, Y. Xu et al., Trigonometric Parallaxes of Massive Star-Forming Regions. VI. Galactic Structure, Fundamental Parameters, and Noncircular Motions, ApJ 700 (July, 2009) 137-148, [0902.3913].

[58] J. P. Vallée, New Velocimetry and Revised Cartography of the Spiral Arms in the Milky Way: A Consistent Symbiosis, AJ 135 (Apr., 2008) 1301-1310.

[59] M. J. Reid, K. M. Menten, A. Brunthaler, X. W. Zheng, T. M. Dame, Y. Xu et al., Trigonometric Parallaxes of High Mass Star Forming Regions: The Structure and Kinematics of the Milky Way, ApJ 783 (Mar., 2014) 130, [14 01.5377$].$

[60] P. J. McMillan and J. J. Binney, The uncertainty in Galactic parameters, MNRAS 402 (Feb., 2010) 934-940, [0 907.4685].

[61] F. Hammer, M. Puech, L. Chemin, H. Flores and M. D. Lehnert, The Milky Way, an Exceptionally Quiet Galaxy: Implications for the Formation of Spiral Galaxies, ApJ 662 (June, 2007) 322-334, [astro-ph/0702585].

[62] D. P. Finkbeiner, T. R. Slatyer, N. Weiner and I. Yavin, PAMELA, DAMA, INTEGRAL and signatures of metastable excited WIMPs, JCAP 9 (Sept., 2009) 037, [0 903.1037$].$

[63] L. D. Anderson and T. M. Bania, Resolution of the Distance Ambiguity for Galactic H II Regions, ApJ 690 (Jan., 2009) 706-719, [0810.5570].

[64] J. S. Urquhart, A. L. Busfield, M. G. Hoare, S. L. Lumsden, R. D. Oudmaijer, T. J. T. Moore et al., The RMS survey. ${ }^{13} \mathrm{CO}$ observations of candidate massive YSOs in the southern Galactic plane, A\&A 474 (Nov., 2007) 891-901.

[65] J. J. Condon, W. D. Cotton, E. W. Greisen, Q. F. Yin, R. A. Perley, G. B. Taylor et al., The NRAO VLA Sky Survey, AJ 115 (May, 1998) 1693-1716.

[66] J. S. Urquhart, M. G. Hoare, C. R. Purcell, S. L. Lumsden, R. D. Oudmaijer, T. J. T. Moore et al., The RMS survey. $6 \mathrm{~cm}$ continuum VLA observations towards candidate massive YSOs in the northern hemisphere, A\&A 501 (July, 2009) 539-551, [0 905.1174 ]. 
[67] L. Moscadelli and M. Catarzi, Time variability of five strong $12 \mathrm{GHz}$ methanol masers., $A \& A S 116$ (Apr., 1996) 211-238.

[68] S. Goedhart, M. C. Langa, M. J. Gaylard and D. J. Van Der Walt, Short-period variability in the Class II methanol maser source G12.89+0.49 (IRAS 18089-1732), MNRAS 398 (Sept., 2009) 995-1010, [0 906.0295 ].

[69] R. P. Norris, A. M. Hopkins, J. Afonso, S. Brown, J. J. Condon, L. Dunne et al., EMU: Evolutionary Map of the Universe, PASA 28 (Aug., 2011) 215-248, [1106.3219].

[70] G. Umana, C. Trigilio, L. Cerrigone, R. Cesaroni, A. A. Zijlstra, M. Hoare et al., The impact of SKA on Galactic Radioastronomy: continuum observations, Advancing Astrophysics with the SKA (AASKA14) (Apr., 2015) 118, [1412.5833].

[71] J. Green, H. J. Van Langevelde, A. Brunthaler, S. Ellingsen, H. Imai, W. H. T. Vlemmings et al., Maser Astrometry with VLBI and the SKA, Advancing Astrophysics with the SKA (AASKA14) (Apr., 2015) 119, [1504.00485].

[72] M. Thompson, H. Beuther, C. Dickinson, J. MOttram, P. Klaassen, A. Ginsburg et al., The ionised,radical and molecular Milky Way: spectroscopic surveys with the SKA, Advancing Astrophysics with the SKA (AASKA14) (Apr., 2015) 126, [1412.5554]. 